\title{
Co-H-structures on equivariant Moore spaces
}

\author{
by \\ Martin A r kowitz (Hanover, N.H.) and \\ Marek Golasińs ki (Toruń)
}

\begin{abstract}
Let $G$ be a finite group, $\mathbb{O}_{G}$ the category of canonical orbits of $G$ and $\boldsymbol{A}: \mathbb{O}_{G} \rightarrow \mathbb{A b}$ a contravariant functor to the category of abelian groups. We investigate the set of $G$-homotopy classes of comultiplications of a Moore $G$-space of type $(\boldsymbol{A}, n)$ where $n \geq 2$ and prove that if such a Moore $G$-space $X$ is a cogroup, then it has a unique comultiplication if $\operatorname{dim} X<2 n-1$. If $\operatorname{dim} X=2 n-1$, then the set of comultiplications of $X$ is in one-one correspondence with $\operatorname{Ext}^{n-1}(\boldsymbol{A}, \boldsymbol{A} \otimes \boldsymbol{A})$. Then the case $G=\mathbb{Z}_{p^{k}}$ leads to an example of infinitely many $G$-homotopically distinct $G$-maps $\varphi_{i}: X \rightarrow Y$ such that $\varphi_{i}^{H}, \varphi_{j}^{H}: X^{H} \rightarrow Y^{H}$ are homotopic for all $i, j$ and all subgroups $H \subseteq G$.
\end{abstract}

1. Introduction. If $A$ is an abelian group and $n$ an integer $\geq 2$, then a Moore space of type $(A, n)$ is a space with a single nonvanishing homology group $A$ in dimension $n$. Moore spaces play a central role in homotopy theory and have been widely studied. In particular, the co-H-structures of a Moore space have been investigated. It is known that for $n>2$ there is a unique co-H-structure (up to homotopy) on a Moore space, but that for $n=2$ there may be several distinct co-H-structures (e.g., see $[A-G]$ ). In this paper we consider these results within the context of equivariant homotopy theory.

Throughout, $G$ denotes a finite group and all spaces, maps, homotopies and actions are pointed. We work in the category $G$-Top of $_{*} G$-spaces which have the $G$-homotopy type of $G$-CW-complexes $[\mathrm{Br}]$. We denote by $\mathbb{O}_{G}$ the category of canonical orbits of $G$ whose objects are the left cosets $G / H$ as $H$ ranges over all subgroups of $G$ and whose morphisms are the equivariant maps $G / H \rightarrow G / K$ with respect to left translation. An $\mathbb{O}_{G}$-module is a contravariant functor from $\mathbb{O}_{G}$ into $\mathbb{A} b$, the category of abelian groups. For a pair $(X, Y)$ of $G$-spaces and an integer $n \geq 1$, an $\mathbb{O}_{G}$-module $\boldsymbol{H}_{n}(X, Y)$ : $\mathbb{O}_{G} \rightarrow \mathbb{A b}$ can be defined as follows: $\boldsymbol{H}_{n}(X, Y)(G / H)=H_{n}\left(X^{H}, Y^{H}\right)$, where $H_{n}$ denotes the $n$th singular homology functor and $X^{H}$ is the $H$ fixedpoint subspace of $X$. Similarly, with $n \geq 3$ ( $n \geq 2$ if $Y$ is the base point $*$ ) we define $\boldsymbol{\pi}_{n}(X, Y): \mathbb{O}_{G} \rightarrow \mathbb{A b}$ using the $n$th homotopy functor $\pi_{n}$. For

1991 Mathematics Subject Classification: 55P45, 55P91, 55U35, 18 G55. 
$Y=*$, these $\mathbb{O}_{G}$-modules are denoted by $\widetilde{\boldsymbol{H}}_{n}(X)$ and $\boldsymbol{\pi}_{n}(X)$, respectively.

Now let $\boldsymbol{A}: \mathbb{O}_{G} \rightarrow \mathbb{A b}$ be an $\mathbb{O}_{G}$-module and $n \geq 2$ an integer. Following Kahn $\left[\mathrm{Ka}_{1}\right]$, we define a Moore $G$-space of type $(\boldsymbol{A}, n)$ to be a $G$-space $X$ such that

(1) $X^{H}$ is 1-connected for all subgroups $H$ of $G$,

(2) $\widetilde{\boldsymbol{H}}_{n}(X) \cong \boldsymbol{A}$ as $\mathbb{O}_{G}$-modules,

(3) $\widetilde{\boldsymbol{H}}_{i}(X)=0$ for $i \neq n$.

If the $H$-fixedpoint sets $X^{H}$ are disregarded when $H$ is a nontrivial subgroup, then we obtain a classical Moore $G$-space. More precisely, if $A$ is a $G$-module and $n \geq 2$, then a classical Moore $G$-space of type $(A, n)$ is a $G$-space $X$ such that

(1) $X$ is 1-connected,

(2) $\widetilde{H}_{n}(X) \cong A$ as $G$-modules,

(3) $\widetilde{H}_{i}(X)=0$ for $i \neq n$.

Moore $G$-spaces have been considered in several papers ([Do 1 , [Do 2$]$, $\left.\left[\mathrm{Ka}_{1}\right],\left[\mathrm{Ka}_{2}\right]\right)$ and shown to be important in equivariant homotopy theory (e.g., the construction of an equivariant homology decomposition $\left[\mathrm{Ka}_{2}\right]$ ). Furthermore, classical Moore spaces have been extensively studied in connection with the Steenrod problem (e.g., $\left.[\mathrm{Ca}],\left[\mathrm{Ka}_{3}\right],[\mathrm{Sm}]\right)$. Unlike the nonequivariant case, Moore $G$-spaces need not exist for any $\mathbb{O}_{G}$-module $\boldsymbol{A}$, and when they exist, they need not be unique (see Section 2 for known existence and uniqueness results). This is so even for classical Moore $G$ spaces.

In this paper we extend the results of $[\mathrm{A}-\mathrm{G}]$ to the equivariant case and investigate the set of $G$-homotopy classes of comultiplications of a Moore $G$-space. We begin with some generalities on closed model categories $\mathbb{C}$. We show that if $X$ is a cogroup object in Ho $\mathbb{C}$, the associated homotopy category of $\mathbb{C}$, then the collection of comultiplications of $X$ is in one-one correspondence with the set of morphisms $\operatorname{Ho} \mathbb{C}(X, F)$, where $F$ is the fibre of the canonical morphism $X \vee X \rightarrow X \times X$. Next we introduce two closed model structures on $G$-Top ${ }_{*}$, one to be used for Moore $G$-spaces and the other for classical Moore $G$-spaces. We then deduce in the next section that a Moore $G$-space $X$ of type $(\boldsymbol{A}, n)$ which is a cogroup has a unique comultiplication if $\operatorname{dim} X<2 n-1$. If $\operatorname{dim} X=2 n-1$, we show that the set of comultiplications of $X$ is in one-one correspondence with $\operatorname{Ext}^{n-1}(\boldsymbol{A}, \boldsymbol{A} \otimes \boldsymbol{A})$. Analogous results are established for classical Moore $G$-spaces. We then apply these considerations to the case $G=\mathbb{Z}_{p^{k}}$. This leads to an example of infinitely many $G$-homotopically distinct $G$-maps $\varphi_{i}: X \rightarrow Y$ such that $\varphi_{i}^{H}, \varphi_{j}^{H}: X^{H} \rightarrow Y^{H}$ are homotopic for all $i, j$ and all subgroups $H \subseteq G$. 
2. Background. The general reference here for category theory is $[\mathrm{Qu}]$. Let $\mathbb{C}$ be a pointed category with finite products and coproducts. For objects $X$ and $Y$ of $\mathbb{C}$, morphisms are written $f: X \rightarrow Y$ or $f \in \mathbb{C}(X, Y)$. In particular, the zero morphism is $0: X \rightarrow Y$ and the identity morphism is $1_{X}: X \rightarrow X$. Let $X \vee Y$ denote the coproduct of $X$ and $Y$ and $X \times Y$ the product of $X$ and $Y$. Then for an object $X$, there is a canonical morphism $j$ : $X \vee X \rightarrow X \times X$ determined by two morphisms $\left(1_{X}, 0\right),\left(0,1_{X}\right): X \rightarrow X \times X$. Let $\triangle=\left(1_{X}, 1_{X}\right): X \rightarrow X \times X$ be the diagonal morphism. A morphism $\varphi: X \rightarrow X \vee X$ such that $j \varphi=\triangle$ is called a comultiplication of $X$, and $X$ is said to have co-structure $\varphi$. If $(1 \vee \varphi) \varphi=(\varphi \vee 1) \varphi: X \rightarrow X \vee X \vee X$ then $\varphi$ is associative. If there exists a morphism $\eta: X \rightarrow X$ such that $\nabla\left(\eta \vee 1_{X}\right) \varphi=\nabla\left(1_{X} \vee \eta\right) \varphi=0: X \rightarrow X$, where $\nabla: X \vee X \rightarrow X$ is the folding morphism, we say that $\eta$ is an inverse. The triple $(X, \varphi, \eta)$ is then called a cogroup object in $\mathbb{C}$. If $(X, \varphi, \eta)$ is a cogroup object in $\mathbb{C}$ and $Y$ is any object, then $\varphi$ and $\eta$ induce a group structure on the set $\mathbb{C}(X, Y)$ such that for every morphism $g: Y \rightarrow Y^{\prime}$, the induced map $g_{*}: \mathbb{C}(X, Y) \rightarrow \mathbb{C}\left(X, Y^{\prime}\right)$ is a homomorphism.

Now let $\mathbb{C}$ be a pointed closed model category. We localize $\mathbb{C}$ with respect to the class of weak equivalences and obtain the homotopy category Ho $\mathbb{C}$ $[\mathrm{Qu}]$. A co-structure on an object in Ho $\mathbb{C}$ is called a co-H-structure and a cogroup object in $\mathrm{Ho} \mathbb{C}$ is called a co-H-group. Quillen $[\mathrm{Qu}]$ has defined a suspension functor $\Sigma$ Ho $\mathbb{C} \rightarrow \mathrm{Ho} \mathbb{C}$ such that $\Sigma X$ is a co-H-group. For any objects $X, Y$ in Ho $\mathbb{C}$, let us denote $\operatorname{Ho} \mathbb{C}(X, Y)$ by $[X, Y]$. Then if $f: X \rightarrow$ $Y$, there exists an object $F$, called the fibre of $f$, such that for any object $Z$, the following sequence is exact $[\mathrm{Qu}]$ :

$$
\ldots \rightarrow[\Sigma Z, X] \stackrel{f_{*}}{\rightarrow}[\Sigma Z, Y] \rightarrow[Z, F] \rightarrow[Z, X] \stackrel{f_{*}}{\rightarrow}[Z, Y] .
$$

Let $X$ be a co-H-group, $\mathcal{C}(X) \subseteq[X, X \vee X]$ the set of co-H-structures of $X$ and $F$ the fibre of the canonical morphism $j: X \vee X \rightarrow X \times X$. Then the set $\mathcal{C}(X)$ is an orbit of the action of the group $[X, F]$ on $[X, X \vee X]$ by (right) translation. So there is, in general, no natural group structure on $\mathcal{C}(X)$. However, if an element of $\mathcal{C}(X)$ is chosen as a base point it is possible to offer a direct interpretation of the group structure of $\mathcal{C}(X)$.

Proposition 2.1. For any co-H-group object $X$ in $\mathrm{Ho} \mathbb{C}$, there is a group isomorphism

$$
\mathcal{C}(X) \stackrel{\simeq}{\rightrightarrows}[X, F]
$$

The proof follows from the above long exact sequence applied to $j$ together with the methods of $[\mathrm{A}-\mathrm{G}]$.

Next let $\mathbb{T o p}_{*}$ be the category of pointed topological spaces. We give $\mathbb{T o p}_{*}$ the structure of a pointed closed model category by defining weak equivalences, fibrations and cofibrations in $\mathbb{T o p}_{*}$ in the usual way [Qu]. Let 
$G$-Top $_{*}$ be the category with objects pointed $G$-spaces and morphisms $G$ maps. We define a closed model category structure $\mathbf{I}$ on $G$-Top t $_{*}$ as follows:

I-1. A $G$-map $f: X \rightarrow Y$ is a weak equivalence if the maps $f^{H}: X^{H} \rightarrow$ $Y^{H}$ of $H$-fixedpoint subspaces are weak equivalences in $\mathbb{T o p}_{*}$ for all subgroups $H \subseteq G$.

I-2. A $G$-map $f: E \rightarrow B$ is a fibration if $f^{H}: E^{H} \rightarrow B^{H}$ are fibrations in $\mathbb{T o p}_{*}$ for all subgroups $H \subseteq G$.

I-3. Cofibrations are determined by weak equivalences and fibrations by means of the lifting property [Qu, p. 5.1].

We also define a second closed model category structure II on $G$-Top : $_{*}$

II-1. A $G$-map $f: X \rightarrow Y$ is a weak equivalence if $f$ is a weak equivalence in $\mathbb{T o p}_{*}$.

II-2. A $G$-map $f: E \rightarrow B$ is a fibration if $f$ is a fibration in $\mathbb{T}_{*}$.

II-3. Cofibrations are determined by weak equivalences and fibrations as above.

One checks that I and II satisfy the axioms for a pointed closed model category (cf. $[\mathrm{D}-\mathrm{D}-\mathrm{K}])$ and thus one obtains homotopy categories

$$
\mathrm{Ho}^{\mathbf{I}} G-\mathbb{T o p}_{*} \quad \text { and } \quad \mathrm{Ho}^{\mathbf{I I}} G-\mathbb{T o p}_{*}
$$

by localizing with respect to the weak equivalences of I and II, respectively.

Finally, we summarize from $\left[\mathrm{Ka}_{1}\right]$ conditions for the existence and uniqueness of a Moore $G$-space $X$ of type $(\boldsymbol{A}, n)$, where $\boldsymbol{A}$ is an $\mathbb{O}_{G}$-module. We are especially interested in when $X$ is a cogroup in the appropriate category. If $\operatorname{proj} \operatorname{dim} \boldsymbol{A} \leq 1$, then a Moore $G$-space $X$ of type $(\boldsymbol{A}, n)$ exists and any two are $G$-equivalent (i.e., equivalent objects in $\operatorname{Ho}^{\mathbf{I}} G-\mathbb{T o p}_{*}$ ). We denote $X$ by $M(\boldsymbol{A}, n)$. Thus, for $\operatorname{proj} \operatorname{dim} \boldsymbol{A} \leq 1, \Sigma M(\boldsymbol{A}, n) \cong M(\boldsymbol{A}, n+1)$. Therefore, a Moore $G$-space of type $(\boldsymbol{A}, n)$ with $n \geq 3$ and $\operatorname{proj} \operatorname{dim} \boldsymbol{A} \leq 1$ is a co-H-group. This is also true for $n=2$. For, following Kahn's methods $\left[\mathrm{Ka}_{1}\right]$, we can find a $G$-space $K$ such that

$$
\widetilde{\boldsymbol{H}}_{i}(K)= \begin{cases}\boldsymbol{A} & \text { for } i=1, \\ 0 & \text { for } i \neq 1 .\end{cases}
$$

By uniqueness, $M(\boldsymbol{A}, 2) \cong \Sigma K$. Therefore, $M(\boldsymbol{A}, n)$ is a cogroup object in $\mathrm{Ho}^{\mathbf{I}} G-\mathbb{T} \mathrm{op}_{*}$ for $n \geq 2$ and $\operatorname{proj} \operatorname{dim} \boldsymbol{A} \leq 1$.

If $A$ is a $G$-module and $\operatorname{proj} \operatorname{dim} A<\infty$, then by $\left[\mathrm{Ka}_{1}\right.$, p. 260] a classical Moore $G$-space of type $(A, n)$ exists and any two are equivalent (i.e., are equivalent objects in $\mathrm{Ho}^{\mathbf{I I}} G$-Top $\left.{ }_{*}\right)$. This is seen by assigning an $\mathbb{O}_{G}$-module $\widetilde{\boldsymbol{A}}$ to $A$ as follows: let $\widetilde{\boldsymbol{A}}(G / H)=0$ for $H \neq E$ and $\widetilde{\boldsymbol{A}}(G / E)=A$, where $E$ is the trivial subgroup of $G$. Then $\operatorname{proj} \operatorname{dim} \widetilde{\boldsymbol{A}} \leq 1$ and the existence of a classical Moore $G$-space follows from the previous paragraph. Uniqueness is also established and one concludes as above that a classical Moore $G$-space 
of type $(A, n)$ with $\operatorname{proj} \operatorname{dim} A<\infty$ and $n \geq 2$ is a cogroup object in $\mathrm{Ho}^{\text {II }} G-\mathbb{T o p}_{*}$.

We next assume that $\boldsymbol{A}$ is a rational $\mathbb{O}_{G}$-module, that is, an $\mathbb{O}_{G}$-module such that each $\boldsymbol{A}(G / H)$ is a vector space over the field $\mathbb{Q}$ of rational numbers. Using the above results and work of [Un], we conclude that a Moore $G$ space of type $(\boldsymbol{A}, n)$ always exists. If, in addition, $\operatorname{proj} \operatorname{dim} \boldsymbol{A}<n$, then all such Moore $G$-spaces are equivalent. Thus if $\boldsymbol{A}$ is a rational $\mathbb{O}_{G}$-module of proj $\operatorname{dim}<n$, the Moore $G$-space of type $(\boldsymbol{A}, n)$ is a cogroup object in $\mathrm{Ho}^{\mathbf{I}} G$ $\mathbb{T o p}_{*}, n \geq 2$. Similar considerations apply to classical Moore $G$-spaces.

3. Comultiplications. In this section we use Proposition 2.1 to determine the set $\mathcal{C}(X)$ of co-H-structures of $X$, where $X$ is a Moore $G$-space of type $(\boldsymbol{A}, n)$, a co-group and $\operatorname{dim} X \leq 2 n-1$. In preparation for this we need some results on Bredon cohomology.

For a given $\mathbb{O}_{G}$-module $\boldsymbol{B}$, Bredon $[\mathrm{Br}]$ and Illman $\left[\mathrm{Il}_{2}\right]$ construct an equivariant cohomology theory $H_{G}^{*}(-, \boldsymbol{B})$ defined on the category of pairs of $G$-spaces and $G$-maps. This cohomology theory satisfies all the EilenbergSteenrod axioms for cohomology suitably interpreted for equivariant spaces and maps. The category of $\mathbb{O}_{G}$-modules (i.e., the category whose objects are $\mathbb{O}_{G}$-modules and whose morphisms are natural transformations) contains sufficiently many projectives and injectives $[\mathrm{Br}]$. Thus one can define $\operatorname{Ext}^{p}$ for this category in the usual way as the right derived functor of the Hom functor.

For a pair $(X, Y)$ of $G-C W$-complexes, Bredon [Br] derives a spectral sequence $\left\{E_{r}^{p, q}\right\}$ with

$$
E_{2}^{p, q}=\operatorname{Ext}^{p}\left(\boldsymbol{H}_{q}(X, Y), \boldsymbol{B}\right) \Rightarrow H_{G}^{p+q}(X, Y ; \boldsymbol{B}) .
$$

There is a decreasing filtration of the group $H^{p+q}=H_{G}^{p+q}(X, Y ; \boldsymbol{B})$,

$$
H^{p+q}=F^{-1} H^{p+q} \supseteq F^{0} H^{p+q} \supseteq \ldots \supseteq F^{p+q} H^{p+q}=0,
$$

with

$$
F^{p} H^{p+q} / F^{p+1} H^{p+q}=E_{\infty}^{p, q} .
$$

Let now $X$ be a Moore $G$-space of type $(\boldsymbol{A}, n)$ for an $\mathbb{O}_{G}$-module $\boldsymbol{A}$ and $n \geq 2$. Then the Bredon spectral sequence degenerates, i.e., $E_{2}^{p, q}=0$ for $p \geq 0$ and $q \neq n$ and $E_{2}^{p, n}=\operatorname{Ext}^{p}(\boldsymbol{A}, \boldsymbol{B})$. Thus

$0=E_{2}^{p, q}=E_{3}^{p, q}=\ldots=E_{\infty}^{p, q} \quad$ for $q \neq n \quad$ and $\quad E_{2}^{p, n}=E_{3}^{p, n}=\ldots=E_{\infty}^{p, n}$. Hence $F^{p-q} H^{p} / F^{p-q+1} H^{p}=E_{\infty}^{p-q, q}=0$ for $q \neq n$ and so (cf. $\left[\mathrm{Ka}_{1}\right]$ )

$$
H_{G}^{p}(X, \boldsymbol{B})=\operatorname{Ext}^{p-n}(\boldsymbol{A}, \boldsymbol{B}) .
$$

For a Moore $G$-space $X$ of type $(\boldsymbol{A}, n)$, let $F$ denote the fibre of the map $j: X \vee X \rightarrow X \times X$ in the category $\mathrm{Ho}^{\mathbf{I}} G-\mathbb{T}$ op $_{*}$ and let $X$ be a cogroup in $\mathrm{Ho}^{\mathbf{I}} G-\mathbb{T o p}_{*}$. We denote by $[-,-]_{G}$ the set of morphisms in $\mathrm{Ho}^{\mathbf{I}} G-\mathbb{T}_{*}$. 
TheOREM 3.2. Under the above assumptions, if $\operatorname{dim} X=d \leq 2 n-1$ then the set $\mathcal{C}(X)$ of co-H-structures of $X$ is in one-one correspondence with the group $\operatorname{Ext}^{d-n}\left(\boldsymbol{A}, \boldsymbol{\pi}_{d}(F)\right)$.

P r o o f. Since $\boldsymbol{H}_{i}(X \times X, X \vee X)=0$ for $i<2 n$ and $\boldsymbol{H}_{2 n}(X \times X, X \vee X)=$ $\boldsymbol{H}_{n}(X) \otimes \boldsymbol{H}_{n}(X)=\boldsymbol{A} \otimes \boldsymbol{A}$, by the Hurewicz theorem, $\boldsymbol{\pi}_{i}(X \times X, X \vee X)=0$ for $i<2 n$ and $\boldsymbol{\pi}_{2 n}(X \times X, X \vee X)=\boldsymbol{A} \otimes \boldsymbol{A}$. Thus $\boldsymbol{\pi}_{i}(F)=0$ for $i<2 n-1$ and $\boldsymbol{\pi}_{2 n-1}(F)=\boldsymbol{A} \otimes \boldsymbol{A}$. Let $F_{d}$ denote the $d$ th term of the Postnikov $G$-tower of the $G$-space $F\left([\mathrm{D}-\mathrm{D}-\mathrm{K}],\left[\operatorname{Tr}_{1}\right]\right)$ and $f_{d}: F \rightarrow F_{d}$ the canonical map. Then the morphism $\boldsymbol{\pi}_{i}(F) \rightarrow \boldsymbol{\pi}_{i}\left(F_{d}\right)$ induced by $f_{d}$ is an isomorphism for $i \leq d$ and epimorphism for $i=d+1$. Since $\operatorname{dim} X=d$, the equivariant Whitehead theorem $\left(\left[\mathrm{Il}_{1}\right]\right.$, [Ma]) implies that $\left(f_{d}\right)_{*}:[X, F]_{G} \rightarrow\left[X, F_{d}\right]_{G}$ is a bijection. But $F_{d} \cong K\left(\boldsymbol{\pi}_{d}(F), d\right)$, the Eilenberg-MacLane space of type $\left(\boldsymbol{\pi}_{d}(F), d\right)$, since $d \leq 2 n-1$. Therefore

$$
[X, F]_{G} \cong\left[X, F_{d}\right]_{G} \cong\left[X, K\left(\boldsymbol{\pi}_{d}(F), d\right)\right]_{G} \cong H_{G}^{d}\left(X, \boldsymbol{\pi}_{d}(F)\right)
$$

and this is $\operatorname{Ext}^{d-n}\left(\boldsymbol{A}, \boldsymbol{\pi}_{d}(F)\right)$ by (3.1). The result now follows from Proposition 2.1.

Corollary 3.3. If $\operatorname{dim} X<2 n-1$, then $\mathcal{C}(X)$ has one element. If $\operatorname{dim} X=2 n-1$, then $\mathcal{C}(X)$ is in one-one correspondence with $\operatorname{Ext}^{n-1}(\boldsymbol{A}$, $\boldsymbol{A} \otimes \boldsymbol{A})$.

Now let $A$ be a $G$-module and $X$ a classical Moore $G$-space of type $(A, n)$ and a cogroup object in the category $\mathrm{Ho}^{\mathbf{I I}} G-\mathbb{T}_{*}$. Then, by Proposition 2.1, $\mathcal{C}(X)$ is in one-one correspondence with the set $[X, F]_{\text {II }}$ of morphisms in $\mathrm{Ho}^{\text {II }} G$-Top of $_{*}$ to $F$, where $F$ is the fibre of $j: X \vee X \rightarrow X \times X$. From the Hurewicz theorem we deduce that $\pi_{i}(F)=0$ for $i<2 n-1$ and $\pi_{2 n-1}(F) \cong A \otimes A$ as $G$-modules. Suppose that $\operatorname{dim} X=d \leq 2 n-1$ and $F_{d}$ is the $d$ th term of the Postnikov $G$-tower of $F$. Then as above $[X, F]_{\mathbf{I I}}$ is in one-one correspondence with $\left[X, F_{d}\right]_{\mathbf{I I}}$ and $F_{d}$ is an Eilenberg-MacLane $G$ space $K\left(\pi_{d}(F), d\right)$. Let $\tilde{\boldsymbol{\pi}}_{d}(F)$ be the $\mathbb{O}_{G}$-module defined by $\widetilde{\boldsymbol{\pi}}_{d}(F)(G / H)$ $=0$ for $H \neq E$ and $\tilde{\pi}_{d}(F)(G / E)=\pi_{d}(F)$, where $E$ is the trivial subgroup of $G$. Then $\left[X, F_{d}\right]_{\mathbf{I I}} \cong\left[X, K\left(\widetilde{\boldsymbol{\pi}}_{d}(F), d\right)\right]$, where $K\left(\widetilde{\boldsymbol{\pi}}_{d}(F), d\right)$ is the Eilenberg-MacLane $G$-space of type $\left(\tilde{\boldsymbol{\pi}}_{d}(F), d\right)$. Hence by $(3.1),[X, F]_{\mathbf{I I}} \cong$ $\operatorname{Ext}_{G}^{d-n}\left(A, \pi_{d}(F)\right)$, where $\operatorname{Ext}_{G}^{p}$ denotes the $p$ th Ext functor in the category of $G$-modules. Thus we obtain

Corollary 3.4. Let $A$ be a $G$-module and $X$ a classical Moore $G$-space of type $(A, n)$ and a cogroup object in $\mathrm{Ho}^{\text {II }} G$-Top ${ }_{*}$. If $\operatorname{dim} X<2 n-1$, then $\mathcal{C}(X)$ has one element. If $\operatorname{dim} X=2 n-1$, then $\mathcal{C}(X)$ is in one-one correspondence with $\operatorname{Ext}_{G}^{n-1}(A, A \otimes A)$.

R e m ark 3.5. Corollary 3.2 (and 3.3) can also be proved by using a spectral sequence derived from an exact couple based on dual Puppe sequences 
obtained from the fibrations $K\left(\boldsymbol{\pi}_{q}, q\right) \rightarrow F_{q} \rightarrow F_{q-1}$ (cf. [M-T, Chap. 14]). In addition, this method shows, under the hypothesis of Corollary 3.3, that if $\operatorname{dim} X>2 n-1$ and $\operatorname{Ext}^{n-1}(\boldsymbol{A}, \boldsymbol{A} \otimes \boldsymbol{A}) \neq 0$, then $\mathcal{C}(X)$ has more than one element.

4. An example. Let $\mathbb{Z}_{p^{k}}$ be the group of integers $\bmod p^{k}$, where $p$ is a prime, and let us denote $\mathbb{O}_{\mathbb{Z}_{p^{k}}}$ by $\mathbb{O}\left(\mathbb{Z}_{p^{k}}\right)$. Any $\mathbb{O}\left(\mathbb{Z}_{p^{k}}\right)$-module $\boldsymbol{A}$ determines a sequence

$$
A_{0} \stackrel{m_{1}}{\rightarrow} A_{1} \stackrel{m_{2}}{\rightarrow} \ldots \stackrel{m_{k}}{\rightarrow} A_{k}
$$

where $A_{i}=\boldsymbol{A}\left(\mathbb{Z}_{p^{k}} / \mathbb{Z}_{p^{k-i}}\right)$ and $m_{i}=\boldsymbol{A}\left(\pi_{i}\right)$, where $\pi_{i}: \mathbb{Z}_{p^{k}} / \mathbb{Z}_{p^{k-i}} \rightarrow$ $\mathbb{Z}_{p^{k}} / \mathbb{Z}_{p^{k-i+1}}$ are projections.

We restrict our considerations to rational $\mathbb{O}\left(\mathbb{Z}_{p^{k}}\right)$-modules, where all $A_{i}$ are $\mathbb{Q}$-vector spaces and all $m_{i}$ are linear maps. Triantafillou $[\operatorname{Tr}]$ shows that for any such $\mathbb{O}\left(\mathbb{Z}_{p^{k}}\right)$-module $\boldsymbol{A}$, proj $\operatorname{dim} \boldsymbol{A} \leq 1$. Furthermore, $\boldsymbol{A}$ is projective if and only if all $m_{i}$ are injections.

We define a rational $\mathbb{O}\left(\mathbb{Z}_{p^{k}}\right)$-module $\boldsymbol{A}$ to be null if all $m_{i}=0$. With such a null $\mathbb{O}\left(\mathbb{Z}_{p^{k}}\right)$-module we associate the commutative diagram

$$
\begin{aligned}
& A_{0} \stackrel{0}{\rightarrow} \quad A_{1} \quad \stackrel{0}{\rightarrow} \quad A_{2} \quad \stackrel{0}{\rightarrow} \quad \ldots \quad \stackrel{0}{\rightarrow} \quad A_{k} \\
& \| \begin{array}{llll}
A_{1} & \uparrow p_{2} & \ldots & \uparrow p_{k}
\end{array} \\
& A_{0} \stackrel{i_{0}}{\rightarrow} A_{0} \oplus A_{1} \stackrel{i_{0,1}}{\rightarrow} A_{0} \oplus A_{1} \oplus A_{2} \stackrel{i_{0,1,2}}{\longrightarrow} \ldots \stackrel{i_{0,1, \ldots, k-1}}{\longrightarrow} A_{0} \oplus \ldots \oplus A_{k} \\
& \uparrow \quad \uparrow i_{0} \quad \uparrow i_{0,1} \quad \ldots \quad \uparrow i_{0,1, \ldots, k-1} \\
& 0 \rightarrow A_{0} \quad \stackrel{i_{0}}{\longrightarrow} \quad A_{0} \oplus A_{1} \quad \stackrel{i_{0,1}}{\longrightarrow} \quad \ldots \stackrel{i_{0,1, \ldots, k-2}}{\longrightarrow} A_{0} \oplus \ldots \oplus A_{k-1}
\end{aligned}
$$

where the arrows represent canonical projections and injections. Here the second horizontal line gives a rational $\mathbb{O}\left(\mathbb{Z}_{p^{k}}\right)$-module $\boldsymbol{P}_{0}$ and the third horizontal line gives a rational $\mathbb{O}\left(\mathbb{Z}_{p^{k}}\right)$-module $\boldsymbol{P}_{1}$ such that

$$
\boldsymbol{P}_{0}\left(\mathbb{Z}_{p^{k}} / \mathbb{Z}_{p^{k-i}}\right)=A_{0} \oplus \ldots \oplus A_{i}=\boldsymbol{P}_{1}\left(\mathbb{Z}_{p^{k}} / \mathbb{Z}_{p^{k-i+1}}\right) .
$$

Since all the maps in $\boldsymbol{P}_{0}$ and $\boldsymbol{P}_{1}$ are injective, $\boldsymbol{P}_{0}$ and $\boldsymbol{P}_{1}$ are projective $\mathbb{O}\left(\mathbb{Z}_{p^{k}}\right)$-modules. Therefore, we have a projective resolution

$$
0 \rightarrow \boldsymbol{P}_{1} \stackrel{d}{\rightarrow} \boldsymbol{P}_{0} \stackrel{\varepsilon}{\rightarrow} \boldsymbol{A} \rightarrow 0 .
$$

If $\boldsymbol{B}$ is another null $\mathbb{O}\left(\mathbb{Z}_{p^{k}}\right)$-module, then the induced map

$$
d^{*}: \operatorname{Hom}\left(\boldsymbol{P}_{0}, \boldsymbol{B}\right) \rightarrow \operatorname{Hom}\left(\boldsymbol{P}_{1}, \boldsymbol{B}\right)
$$

is zero. Hence

$$
\operatorname{Ext}^{1}(\boldsymbol{A}, \boldsymbol{B})=\operatorname{Hom}\left(\boldsymbol{P}_{1}, \boldsymbol{B}\right) / \operatorname{Im} d^{*}=\operatorname{Hom}\left(\boldsymbol{P}_{1}, \boldsymbol{B}\right)=\bigoplus_{i=0}^{n-1} \operatorname{Hom}\left(A_{i}, B_{i+1}\right) .
$$

Thus we have proved 
Proposition 4.1. If $\boldsymbol{A}$ and $\boldsymbol{B}$ are null $\mathbb{O}\left(\mathbb{Z}_{p^{k}}\right)$-modules then

$$
\operatorname{Ext}^{1}(\boldsymbol{A}, \boldsymbol{B})=\bigoplus_{i=0}^{k-1} \operatorname{Hom}\left(A_{i}, B_{i+1}\right) \text {. }
$$

This leads to the following example.

EXAMPLE 4.2. For the group $G=\mathbb{Z}_{p^{k}}$, there are $G$-spaces $X$ and $Y$ and $G$-maps $\varphi_{i}: X \rightarrow Y, i=1,2, \ldots$, such that $\varphi_{i}$ and $\varphi_{j}$ are not $G$-homotopic for all $i \neq j$ and $\varphi_{i}^{H}, \varphi_{j}^{H}: X^{H} \rightarrow Y^{H}$ are homotopic for all $i, j$ and all subgroups $H$ of $G$.

For this example we let $\boldsymbol{A}$ be a null $\mathbb{O}\left(\mathbb{Z}_{p^{k}}\right)$-module such that $\operatorname{Hom}\left(A_{i}\right.$, $\left.A_{i+1} \otimes A_{i+1}\right) \neq 0$ for some $i \in\{0,1, \ldots, k-1\}$, for example, $A_{i}=A_{i+1}=\mathbb{Q}$. Since $\operatorname{proj} \operatorname{dim} \boldsymbol{A} \leq 1$, there is a Moore $G$-space $X$ of type $(\boldsymbol{A}, 2)$ which is a co-H-group (see Section 2). Kahn $\left[\mathrm{Ka}_{1}\right.$, p. 259] has shown how to construct $X$ such that $\operatorname{dim} X=3$. By Corollary $3.3, \mathcal{C}(X)$ is in one-one correspondence with $\operatorname{Ext}^{1}(\boldsymbol{A}, \boldsymbol{A} \otimes \boldsymbol{A})$. By Proposition 4.1, this latter group is isomorphic to $\bigoplus_{i=1}^{n-1} \operatorname{Hom}\left(A_{i}, A_{i+1} \otimes A_{i+1}\right) \neq 0$. Thus $\mathcal{C}(X)$ is an infinite set and so there are infinitely many co-H-structures $\varphi_{i}: X \rightarrow X \vee X=Y$ in

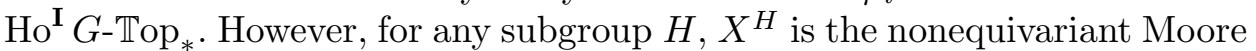
space of type $(\boldsymbol{A}(G / H), 2)$ and each $\varphi_{i}^{H}$ is a comultiplication of $X^{H}$. But by $[\mathrm{A}-\mathrm{G}]$ the comultiplications of $X^{H}$ are in one-one correspondence with $\operatorname{Ext}(\boldsymbol{A}(G / H), \boldsymbol{A}(G / H) \otimes \boldsymbol{A}(G / H))$. This group is trivial since $\boldsymbol{A}(G / H)$ is a $\mathbb{Q}$-vector space. Thus for each subgroup $H$ of $G, \varphi_{i}^{H}$ is homotopic to $\varphi_{j}^{H}$ for all $i, j=1,2, \ldots$

Finally, we close with a problem suggested by $[\mathrm{A}-\mathrm{G}]$. Given an action of a finite group $G$ on $\mathbb{Z}_{m}$, the integers $\bmod m$. Suppose there is a classical Moore $G$-space $X$ of type $\left(\mathbb{Z}_{m}, 2\right)$ which is a co-H-group.

Problem 4.3. Describe the set $\mathcal{C}(X)$ of all comultiplications of $X$.

\section{References}

[A-G] M. Arkowitz and M. Golasiński, Co-H-structures on Moore spaces of type $(A, 2)$, Canad. J. Math., to appear.

[Br] G. E. Bredon, Equivariant Cohomology Theories, Lecture Notes in Math. 34, Springer, 1967.

[Ca] G. Carlsson, A counterexample to a conjecture of Steenrod, Invent. Math. 64 (1981), 171-174.

[Co] S. R. Costenoble and S. W aner, A nonexistence result for Moore G-spectra, Proc. Amer. Math. Soc. 113 (1991), 265-274.

[Do 1 R. Doman, Non-G-equivalent Moore G-spaces of the same type, ibid. 103 (1988), 1317-1321.

[Do 2$] \quad$ - Moore G-spaces which are not co-Hopf G-spaces, Canad. Math. Bull. 32 (1989), 365-368. 
[D-D-K] E. Dror, Dwyer and D. M. Kan, Equivariant maps which are self homotopy equivalences, Proc. Amer. Math. Soc. 80 (1980), 670-672.

[El] A. D. Elmendorf, Systems of fixed point sets, Trans. Amer. Math. Soc. 277 (1983), 275-284.

$\left[\mathrm{Il}_{1}\right]$ S. Illman, Equivariant algebraic topology, Ph.D. Thesis, Princeton University, Princeton, N.J., 1972.

$\left[\mathrm{Il}_{2}\right] \quad$ - Equivariant singular homology and cohomology I, Mem. Amer. Math. Soc. $156(1975)$.

[Ka 1 ] P. J. Kahn, Rational Moore G-spaces, Trans. Amer. Math. Soc. 298 (1986), 245-271.

[Ka2] -, Equivariant homology decompositions, ibid., 273-287.

[Ka3] - Steenrod's problem and $k$-invariants of certain classifying spaces, in: Algebraic $K$-Theory, Lecture Notes in Math. 967, Springer, 1982, 195-214.

[Ma] T. Matumoto, On G-CW complexes and a theorem of J. H. C. Whitehead, J. Fac. Sci. Univ. Tokyo 18 (1971), 363-374.

[M-T] R. E. Mosher and M. C. Tangora, Cohomology Operations and Applications in Homotopy Theory, Harper \& Row, New York, 1968.

[Qu] D. G. Quillen, Homotopical Algebra, Lecture Notes in Math. 43, Springer, 1967.

[Sm] J. R. Smith, Equivariant Moore spaces II-The low dimensional case, J. Pure Appl. Algebra 36 (1985), 187-204.

[Tr] G. V. Triantafillou, Rationalization of Hopf G-spaces, Math. Z. 182 (1983), $485-500$

[Un] H. Unsöld, Topological minimal algebras and Sullivan-de Rham equivalence, Astérisque 113-114 (1984), 337-343.

DEPARTMENT OF MATHEMATICS

DARTMOUTH COLLEGE

HANOVER, NEW HAMPSHIRE 03755

U.S.A.
FACULTY OF MATHEMATICS AND INFORMATICS NICHOLAS COPERNICUS UNIVERSITY

CHOPINA $12 / 18$ 87-100 TORUŃ, POLAND

Received 27 July 1993;

in revised form 21 January 1994 